\title{
Lenalidomide for the Treatment of Low- or Intermediate-1-Risk Myelodysplastic Syndromes Associated with Deletion 5q Cytogenetic Abnormality: An Evidence Review of the NICE Submission from Celgene
}

\author{
Hedwig M. Blommestein ${ }^{1} \cdot$ Nigel Armstrong ${ }^{2} \cdot$ Steve Ryder $^{2} \cdot$ Sohan Deshpande $^{2}$. \\ Gill Worthy $^{2} \cdot$ Caro Noake $^{2} \cdot$ Rob Riemsma $^{2} \cdot$ Jos Kleijnen $^{2,3} \cdot$ Johan L. Severens $^{1}$. \\ Maiwenn $\mathbf{J} . \mathbf{A l}^{1}$
}

Published online: 28 August 2015

(c) The Author(s) 2015. This article is published with open access at Springerlink.com

\begin{abstract}
The National Institute for Health and Care Excellence (NICE) invited the manufacturer of lenalidomide (Celgene) to submit evidence of the clinical and cost effectiveness of the drug for treating adults with myelodysplastic syndromes (MDS) associated with deletion 5q cytogenetic abnormality, as part of the Institute's single technology appraisal (STA) process. Kleijnen Systematic Reviews Ltd (KSR), in collaboration with Erasmus University Rotterdam, was commissioned to act as the Evidence Review Group (ERG). This paper describes the company's submission, the ERG review, and the NICE's subsequent decisions. The ERG reviewed the evidence for clinical and cost effectiveness of the technology, as submitted by the manufacturer to the NICE. The ERG searched for relevant additional evidence and validated the manufacturer's decision analytic model to examine the
\end{abstract}

Hedwig M. Blommestein

blommestein@bmg.eur.nl

1 Department of Health Policy and Management, Institute for Medical Technology Assessment, Erasmus University, Room No. J8-23, PO Box 1738, 3000 DR Rotterdam, The Netherlands

2 Kleijnen Systematic Reviews Ltd, Unit 6, Escrick Business Park, Escrick, York YO19 6FD, UK

3 School for Public Health and Primary Care, Maastricht University, Maastricht, The Netherlands robustness of the cost-effectiveness results. Clinical effectiveness was obtained from a three-arm, European, randomized, phase III trial among red blood cell (RBC) transfusion-dependent patients with low-/intermediate-1risk del5q31 MDS. The primary endpoint was RBC independence for $\geq 26$ weeks, and was reached by a higher proportion of patients in the lenalidomide 10 and $5 \mathrm{mg}$ groups compared with placebo (56.1 and 42.6 vs $5.9 \%$, respectively; both $p<0.001$ ). The option of dose adjustments after 16 weeks due to dose-limiting toxicities or lack of response made long-term effectiveness estimates unreliable, e.g. overall survival (OS). The de novo model of the manufacturer included a Markov state-transition cost-utility model implemented in Microsoft Excel. The base-case incremental cost-effectiveness ratio (ICER) of the manufacturer was $£ 56,965$. The ERG assessment indicated that the modeling structure represented the course of the disease; however, a few errors were identified and some of the input parameters were challenged. In response to the appraisal documentation, the company revised the economic model, which increased the ICER to $£ 68,125$ per quality-adjusted life-year. The NICE Appraisal Committee (AC) did not recommend lenalidomide as a cost-effective treatment. Subsequently, the manufacturer submitted a Patient Access Scheme (PAS) that provided lenalidomide free of charge for patients who remained on treatment after 26 cycles. This PAS improved the ICER to $£ 25,300$, although the AC considered the proportion of patients who received treatment beyond 26 cycles, and hence the ICER, to be uncertain. Nevertheless, the AC accepted a commitment from the manufacturer to publish, once available, data on the proportion of patients eligible for the PAS, and believed this provided reassurance that lenalidomide was a cost-effective treatment for low- or intermediate-1-risk MDS patients. 


\section{Key Points for Decision Makers}

Treatment with lenalidomide is clinically effective because it is associated with improved transfusion independence and health-related quality of life.

Due to the crossover design of the trial after 16 weeks, long-term effectiveness estimates were unreliable and overall survival (OS) was estimated through an indirect relationship, i.e. lenalidomide reduces transfusion dependence and patients becoming transfusion independent have better OS.

The patient access scheme increased uncertainty around the value of the resulting ICER since it only benefits patients on treatment beyond 26 cycles and there was uncertainty on this proportion.

Lenalidomide was accepted under the commitment of the manufacturer to collect data on the proportion of patients on treatment beyond 26 cycles. Data collection by the manufacturer ensures uncertainties can be addressed when the guidance is reviewed; however, it does not address any loss in health benefits for other patients in the National Health Service (NHS) if it turns out that survival has been overestimated and that lenalidomide was in fact not cost effective.

\section{Introduction}

The National Institute for Health and Care Excellence (NICE) is an independent organization providing national guidance on promoting good health and preventing and treating ill health [1]. The single technology appraisal (STA) process is designed to provide recommendations and guidance on a single product, device or other technology with a single indication. The process covers new technologies and enables the NICE to produce guidance shortly after the technology is introduced in the UK. The NICE Appraisal Committee (AC) obtains relevant evidence from several sources: the manufacturer's submission (MS), a report from the appointed independent Evidence Review Group (ERG) and advice from consultees (i.e. experts and other stakeholders). The MS includes a written report and a mathematical model that describe the clinical and cost effectiveness of the technology under investigation. The ERG, an external organization independent of the NICE, reviews the MS and produces a report. After consideration of all the relevant evidence, the AC formulates preliminary guidance in the form of the Appraisal Consultation
Document (ACD) as to whether to recommend the intervention. The stakeholders are invited to comment on this ACD and the submitted evidence. A subsequent ACD may be produced or a Final Appraisal Determination (FAD) issued. The submission of a Patient Access Scheme (PAS) is allowed in order to allow the NICE to recommend treatments that would otherwise not have been found to be cost effective. The PAS is a means of reducing the price of the drug by some means, e.g. simple discount or other formula, and has to be agreed by the Department of Health. This paper presents a summary of the ERG report and the development of NICE guidance based on the findings of the AC for the STA of lenalidomide for treating myelodysplastic syndromes (MDS) associated with deletion $5 \mathrm{q}$ (del5q) cytogenetic abnormality. Full details of all the relevant appraisal documents can be found on the NICE website [2]. This is one in a series of STA summaries being published in Pharmacoeconomics [3-8].

\section{The Decision Problem}

MDS are a heterogeneous group of hematological disorders in which the bone marrow functions abnormally, causing peripheral blood cytopenia due to insufficient production of mature blood cells [9]. MDS can affect red blood cells (RBCs), white blood cells (WBCs) and platelets, resulting in anemia, increase in bleeding, infection and disease transformation to acute myeloid leukemia (AML) [10]. The quality of life of patients with MDS is impaired due to symptoms such as fatigue and dyspnea as well as treatments involving hospitalizations with drug administration and blood transfusions. As reported in 2003, the incidence is approximately 4 per 100,000 population but rises to $>30$ per 100,000 in the over 70 years age group [11]. In the UK, there are approximately 11,200 patients diagnosed with MDS [12], a condition that is mainly caused by cytogenetic abnormalities found in marrow cells. The most common cytogenetic abnormality, present in approximately $15 \%$ of patients with MDS, is del5q [13].

Currently, there is no active treatment available for patients with MDS del5q since stem cell transplantations or treatment with azacitidine are not recommended for this patient group [11]. Patients receive best supportive care (BSC), which includes blood transfusions to control symptoms associated with bone marrow failure and antibiotics to treat or prevent infection. In addition, growth factors such as erythropoietin and/or granulocyte-colony stimulating factors to stimulate the production of RBCs and WBCs are prescribed.

Lenalidomide was already available in the UK for the treatment of relapsed refractory multiple myeloma. In 2013 the European Medicines Agency extended the market 
authorization of lenalidomide to include patients with transfusion-dependent anemia due to low- or intermediate1-risk MDS associated with del5q when other therapeutic options were insufficient or inadequate [14]. Lenalidomide is an oral therapy that aims to reverse transfusion dependence.

The NICE developed a scope for the assessment of lenalidomide, which specified that the clinical and cost effectiveness of this drug should be established, relative to BSC for the treatment of patients with transfusion-dependent anemia due to low- or intermediate-1-risk MDS associated with del5q cytogenetic abnormality with or without other cytogenetic abnormalities.

\section{The Independent Evidence Review Group Review}

Kleijnen Systematic Reviews Ltd (KSR), in collaboration with Erasmus University Rotterdam, acted as the ERG. The ERG reviewed the evidence on the product's clinical and cost effectiveness among low- or intermediate-1-risk MDS del5q patients as submitted by the manufacturer (Celgene). The review embodied three aims:

- to assess whether the MS conformed to the methodological guidelines issued by the NICE [1];

- to assess whether the manufacturer's interpretation and analysis of the evidence was appropriate;

- to indicate the presence of other sources of evidence or alternative interpretations of the evidence that could help to inform NICE guidance.

The ERG critically reviewed the MS, conducted additional searches, explored the impact of assumptions on the incremental cost-effectiveness ratio (ICER), revised the economic model and explored additional scenario analyses. The ERG review detailed here relates to the evidence contained in the original MS and additional information submitted by the manufacturer in response to the clarification questions and $\mathrm{ACD}$, which included a PAS.

\subsection{Clinical Evidence}

The MS included a systematic review of the literature on the clinical effectiveness of lenalidomide. Evidence on the efficacy of lenalidomide was extracted from the MDS-004 trial, a phase III, multicenter, randomized, double-blind, placebo-controlled study [15]. Adult patients with low- or intermediate-1-risk MDS with del5q, with or without additional cytogenetic abnormalities and RBC transfusiondependent anemia $(N=205)$ were randomly assigned to three arms: lenalidomide $10 \mathrm{mg}$ on days $1-21$, lenalidomide $5 \mathrm{mg}$ on days $1-28$, or placebo on days $1-28$ for each 4-week cycle. BSC included blood transfusions that were provided to all transfusion-dependent patients as required. If dose-limiting toxicities occurred, the dose of lenalidomide was reduced. Crossover was allowed at 16 weeks if at least a minor erythroid response (i.e. a $50 \%$ decrease in transfusion requirements) was not achieved, and all but 11 patients on the placebo arm crossed over to lenalidomide $5 \mathrm{mg}$. Before crossover at 16 weeks, two patients $(3 \%)$ in the placebo group, two $(2.9 \%)$ in the lenalidomide $5 \mathrm{mg}$ group and none in the lenalidomide $10 \mathrm{mg}$ group progressed to AML. The primary endpoint was RBC transfusion independence for $\geq 26$ weeks, which was reached in $56.1,42.6$, and $5.9 \%$ of patients in the lenalidomide $10 \mathrm{mg}$, lenalidomide $5 \mathrm{mg}$, and placebo groups, respectively. Transfusion-independent rates in both lenalidomide groups were different compared with placebo $(p<0.001)$. Median duration of transfusion independence was not reached in either lenalidomide group after a median follow up of 1.55 years. Of the patients who initially received placebo and crossed over to lenalidomide $5 \mathrm{mg}, 30.4 \%$ progressed to AML, compared with $23.2 \%$ in the $5 \mathrm{mg}$ group and $21.7 \%$ in the $10 \mathrm{mg}$ group. Median overall survival (OS) was not statistically significantly different between the groups, and ranged from 35.5 to 44.5 months.

Significantly higher proportions of treatment-emergent adverse events (AEs) were reported among patients treated with lenalidomide compared with placebo-treated patients. At least one drug-related $\mathrm{AE}$ was reported in $42 \%$ of the placebo group, $87 \%$ in the lenalidomide $5 \mathrm{mg}$ group and $90 \%$ in the lenalidomide $10 \mathrm{mg}$ group. The most frequent drug-related AEs were neutropenia (15\% in the placebo group and $74 \%$ in each lenalidomide group) and thrombocytopenia ( $2 \%$ in the placebo group and 32 and $36 \%$ in the $5 \mathrm{mg}$ and $10 \mathrm{mg}$ groups, respectively).

Health-related quality of life (HRQoL) outcomes were assessed during the MDS-004 trial using the Functional Assessment of Cancer Therapy-Anemia (FACT-An) questionnaire, which was administered at baseline, and weeks 12, 24, 36 and 48. The EQ-5D was administered at baseline only. Compared with placebo, treatment with lenalidomide was associated with improvements in HRQoL (FACT-An scores) during the initial 12 weeks of therapy. Improved HRQoL was maintained in patients who remained on double-blind treatment with lenalidomide. Among patients who switched from placebo to the lenalidomide $5 \mathrm{mg}$ group, improved HRQoL was observed.

\subsection{Critique of the Clinical Evidence and Interpretation}

According to the ERG, some of the literature searches of the manufacturer were unnecessarily restrictive. For AEs, other study designs could have been included and longer- 
term data could have been sought. It was also not clear how studies were identified for inclusion. Nevertheless, the ERG was unaware of any relevant trials that had been missed, and agreed with the manufacturer that the MDS004 trial was most likely the best source of clinical evidence for the effectiveness of lenalidomide versus BSC.

Data extraction from the MDS-004 trial was reported for the intention-to-treat (ITT) population $(N=205)$ as well as for the modified ITT (mITT) population $(N=139)$. The primary reason for exclusion from the mITT was an inadequate bone marrow sample, preventing confirmation of the diagnosis of low- or intermediate-1-risk MDS del5q. The manufacturer considered the ITT to match more closely with the daily practice population as defined in the NICE scope; therefore the rates from this population were used in the health economic model. Response rates for lenalidomide were based on the lenalidomide $10 \mathrm{mg}$ group $(60.9 \%)$, while the response rate in the model was $7.5 \%$ for the placebo group. Nevertheless, the ITT population included patients not fulfilling the inclusion criteria, i.e. del5q mutation and bone marrow morphology.

Although serious infections were explicitly mentioned as a relevant outcome in the NICE scope, reporting on this outcome in the MS was minimal. Additional data were obtained from the clinical study report and showed that serious infections occurred in the lenalidomide groups twice as often as in the placebo group.

Due to the crossover design after 16 weeks, and dose reductions of the trial, the chances of detecting attributable prolonged survival or acceleration of leukemia progression were limited.

\subsection{Cost Effectiveness}

The manufacturer submitted a de novo economic evaluation on the cost effectiveness of lenalidomide compared with BSC in low- or intermediate-1-risk MDS del5q patients who were transfusion-dependent. An Excel-based Markov model was developed with 14 health states that reflected transfusion requirements, iron chelation, progression to AML, and complications associated with both transfusion dependency and iron chelation therapy. Patients responding to treatment became transfusion-independent, while non-responders remained transfusion-dependent. As a simplifying assumption, all trial patients who responded, regardless of timing, were classed as responders from cycle 1 onwards. Transition probabilities for OS and progression to AML were assumed to be different for transfusion-dependent and -independent patients, and estimated based on the initial response for lenalidomide and BSC of the MDS-004 trial. Response rates for iron chelation therapy and iron overload complication rates were based on the literature [16, 17].
Lenalidomide treatment (plus BSC) is compared with $\mathrm{BSC}$, which was also the comparator in the MDS-004 trial. However, BSC in the trial consisted of blood transfusions only (plus chelation therapy when iron overload occurred), whereas BSC in the UK may also include the provision of erythropoietin-stimulating agents (ESA) or ESA plus granulocyte-colony stimulating factors (G-CSF). The proportion of patients receiving ESA in the model was calculated from the proportion of UK patients in the MDS-004 trial who received ESA prior to the trial, i.e. $28 \%$. Of the side effects associated with lenalidomide, only neutropenia and thrombocytopenia were included in the model since only these were considered serious enough by the manufacturer to warrant inclusion while also being different between the placebo and lenalidomide arms in the trial. Iron chelation is initiated to avoid complications associated with iron overload for transfusion-dependent patients. In the de novo model, patients received either desferrioxamine or deferasirox as iron chelation therapy. Since the number of patients included in the trial was insufficient to obtain transition probabilities for AML mortality, transition probabilities were obtained from the adverse risk group in the article by Wahlin et al. [18]. No half-cycle correction was applied. The model had a National Health Service (NHS) perspective and time horizon of 20 years. Costs and effects were discounted at an annual rate of $3.5 \%$.

During the MDS-004 trial, quality of life was measured using the FACT-An at baseline and in weeks 12, 24, 36 and 48. However, the EQ-5D was measured at baseline only (i.e. when all patients were still transfusion-dependent) and therefore EQ-5D data for transfusion-independent patients were not available. Therefore, utility values were obtained from the study by Szende et al. [19].

Drug acquisition prices were obtained from the British National Formulary (6 March 2013), while the frequency of monitoring associated with the initiation of lenalidomide treatment was based on the summary of product characteristics. Monitoring visits were assumed to occur with a general practitioner (GP). Costs for lenalidomide were based on the dosing observed in the MDS-004 trial and manufacturer's price quotations. An arbitrary standard error of $10 \%$ of the mean was assigned to those cost estimates without a standard error.

The base-case ICER (cost per quality-adjusted life-year [QALY] gained) was $£ 56,965$ per QALY gained. The probabilistic sensitivity analysis (PSA) showed a $0 \%$ probability of the ICER being below $£ 30,000$ per QALY gained. Sensitivity analysis revealed that utility values, the proportion of patients experiencing dose interruptions, and the curve fitting for progression to AML and overall mortality were key parameters. 


\subsection{Critique of the Cost-Effectiveness Evidence and Interpretation}

The economic model described in the MS was considered, by the ERG, to meet the NICE reference case to a reasonable extent and was in line with the decision problem specified in the scope. However, the manufacturer's description of the model did not match their own presented figure. The illogical (e.g. from health-state chelation failure to no chelation cardiac disease) and missing transitions (e.g. from health-state transfusion-dependent chelation to transfusion-independent) were corrected by the ERG.

The ERG challenged some of the assumptions of the manufacturer and therefore made the following adjustments in the ERG base-case.

- A half-cycle correction was implemented since the first few cycles showed a very significant redistribution of patients over the various health states.

- Deferiprone, a third option for chelation therapy, was included in the model (Table 1). This slightly changed the costs per cycle for chelation therapy (from $£ 1383$ to $£ 1332$ ) but also influenced the QALYs since adding this third option increases the proportion of patients receiving oral instead of intravenous chelation therapy (from 71 to $94.3 \%$ ).

- Standard errors without a standard deviation estimate were increased from 10 to $20 \%$ of the mean for adverse events and complications. The standard errors of $10 \%$ were considered too small by the ERG since more variation for costs is usually observed.
- A programming error for the initial response rate for BSC was corrected.

- The effect of G-CSF, in addition to ESA, for nonresponders to BSC was added. The initial response rate was used in the model of the manufacturer. G-CSF is only added to ESA for patients who do not respond to ESA. As a consequence, the model of the manufacturer did not include the effect of G-CSF for nonresponders to BSC.

The revised base-case ICER was $£ 62,674$ per QALY gained. The PSA results showed a $0 \%$ probability that the ICER was below $£ 30,000$ per QALY gained.

\subsubsection{Remaining Concerns}

Utilities were obtained from a study that included broad health-state descriptions covering a range of health problems [19]. The manufacturer assumed that these descriptions adequately described the difference between the transfusion-independent and -dependent health states. However, this was challenged by the ERG. In addition, the ERG raised questions on the assumption of similar utility values for transfusion-dependent and AML health states. The latter was accepted by the ERG since the impact of the utility value assigned to AML was minimal. Moreover, a reasonable alternative for health-state utility values was unavailable.

While the ERG was not entirely convinced that the definition of BSC fully reflects BSC within the NHS, the model outcomes were not very sensitive to changes in the proportion of ESA use.

Table 1 Revised base-case cost-effectiveness analysis, incorporating corrections and amendments identified by the ERG and AC

\begin{tabular}{|c|c|c|c|c|c|c|c|}
\hline & \multicolumn{2}{|l|}{ BSC } & \multicolumn{2}{|c|}{ Lenalidomide } & \multicolumn{2}{|c|}{ Incremental } & \multirow{2}{*}{$\begin{array}{l}\text { ICER } \\
\text { Cost per QALY } \\
\text { gained }(\mathfrak{f})\end{array}$} \\
\hline & Cost $(£)$ & QALY & Cost $(£)$ & QALY & Cost $(£)$ & QALY & \\
\hline Manufacturer's base-case analysis & 105,726 & 2.58 & 156,308 & 3.46 & 50,582 & 0.89 & 56,965 \\
\hline Corrected confirmed programming errors & 104,753 & 2.59 & 156,308 & 3.46 & 51,555 & 0.87 & 59,196 \\
\hline Correcting programming errors dose reduction & 104,753 & 2.59 & 162,628 & 3.46 & 57,875 & 0.87 & 66,453 \\
\hline Additional cycle added & 104,753 & 2.59 & 162,628 & 3.46 & 57,875 & 0.87 & 66,453 \\
\hline Half-cycle correction & 104,052 & 2.57 & 160,343 & 3.43 & 56,292 & 0.87 & 64,929 \\
\hline Chelation therapy deferiprone added & 102,270 & 2.64 & 158,890 & 3.49 & 56,620 & 0.85 & 66,346 \\
\hline Cost AML-adjusted & 100,655 & 2.64 & 157,227 & 3.49 & 56,572 & 0.85 & 66,289 \\
\hline $\begin{array}{l}\text { Response over time (mortality based on } \\
\text { maximum response) }\end{array}$ & 102,839 & 2.64 & 153,817 & 3.45 & 50,978 & 0.81 & 62,773 \\
\hline Cost AEs adjusted & 102,836 & 2.64 & 153,733 & 3.45 & 50,898 & 0.81 & 62,674 \\
\hline ERG revised base-case & 102,836 & 2.64 & 153,733 & 3.45 & 50,898 & 0.81 & 62,674 \\
\hline $\begin{array}{l}\text { Scenario: Monitoring would be undertaken by a } \\
\text { hematologist and progression to AML similar } \\
\text { for lenalidomide and BSC }\end{array}$ & 123,241 & 2.95 & 172,307 & 3.67 & 49,065 & 0.72 & 68,125 \\
\hline
\end{tabular}

$E R G$ Evidence Review Group, $A C$ Appraisal Committee, ICER incremental cost-effectiveness ratio, $Q A L Y$ quality-adjusted life-year, $A M L$ acute myeloid leukemia, AEs adverse events, $B S C$ best supportive care 
Additional scenarios were explored by the ERG and these revealed that utility and cost parameters related to AML complications and AEs had little to no effect on the ICER. The assumption that monitoring occurred with a GP was challenged by the ERG and therefore adjusted to monitoring by a hematologist in an additional scenario analysis, together with revised progression rates to AML (Table 1).

\subsection{Conclusion of the Evidence Review Group (ERG) Report (Before Implementation of the Patient Access Scheme [PAS])}

According to the ERG, there were two main problems with the clinical effectiveness data obtained from the MDS-004 trial and described by the manufacturer in the MS. First, the possibility of crossover after 16 weeks meant that most long-term effectiveness data were unreliable. Second, data were reported for two populations-the ITT and an mITT - and it is not clear how differences between these populations influenced results.

The manufacturer base-case ICER was $£ 56,965$ per QALY gained, while the ERG base-case, correcting for the various issues identified, estimated an ICER of $£ 62,674$ per QALY gained.

\subsection{ERG Research Recommendations}

The ERG concluded that further comparisons of lenalidomide and BSC are required in terms of long-term effectiveness, OS, AML progression and incidence of adverse events. The study on which utilities for the transfusionrelated health states was based did not conform to the NICE reference case. In order to increase the robustness of the health economic outcome, a quality-of-life study among MDS patients would be of great value. Ideally, such a study would ask transfusion-dependent patients, as well as patients who have become transfusion-independent, to fill out the EQ-5D, after which outcomes would be valued using the UK tariff, which is based on the general population [20].

\section{Key Methodological Issues}

Long-term effectiveness, including survival and progression to AML, was compromised by the crossover design of the trial at 16 weeks. The manufacturer stated that survival of patients with MDS is strongly related to transfusion dependency. In order to perform a life-time cost-effectiveness analysis, the model linked OS and progression to AML to transfusion dependency. Therefore, separate timeto-event curves for people who were transfusion-dependent or -independent at 8 weeks were estimated from the data of the MDS-004 trial.

The relationship between survival and transfusion dependency was supported both by data from the MDS-004 trial (achieving transfusion independence was associated with a significant reduction in the risk of death [hazard ratio $0.53 ; 95 \%$ CI $0.31-0.90 ; p=0.019]$ ) and the literature [21, 22].

Utility values used in the model were not obtained according to NICE guidelines and, consequently, the committee needed to decide whether these were acceptable. The STA described here highlights the difficulties of relying on a single randomized controlled trial with a crossover design after 16 weeks. The key issue for a decision maker is whether or not these clinical and economic uncertainties cast sufficient doubt on any patient gain from taking the drug.

\section{National Institute for Health and Care Excellence Guidance}

\subsection{Key Issues Considered by the Appraisal Committee}

Regarding effectiveness, the committee concluded that, on the basis of evidence on transfusion independence and HRQoL, lenalidomide is a clinically effective treatment for people with MDS associated with a del5q cytogenetic abnormality, although uncertainty about whether lenalidomide improved survival remained. The committee concluded that the serious adverse events associated with lenalidomide could be partly managed by a reduction in dose.

According to the committee, cost-effectiveness estimates from the model were uncertain due to uncertainty in the survival estimates. In addition, there was uncertainty as to whether lenalidomide changed the rate of progression to AML. The committee accepted the utility values reported by Szende et al. [19] after consulting the patient expert. In addition, the committee agreed with the adjustments of the ERG and considered that if the model applied similar rates of progression to AML for both treatment groups then the ICER would most plausibly exceed $£ 70,000$ per QALY gained.

\subsubsection{Preliminary Guidance (First Appraisal Consultation Document [ACD])}

After considering the initial evidence submitted by the manufacturer, the ERG report and the testimony of experts and other stakeholders, the AC concluded that lenalidomide could not be recommended for treating transfusion- 
dependent anemia caused by low- or intermediate-1-risk MDS associated with a del5q cytogenetic abnormality when other treatments failed.

\subsubsection{Response to Preliminary Guidance (First ACD) and Additional Analysis Submitted by the Manufacturer}

The manufacturer performed a systematic literature review (July 2013) to better highlight the association between transfusion independence and survival. The AZA-001 trial [23], which demonstrated improved survival after becoming transfusion-independent, was considered most convincing by the ERG. Overall, the committee concluded that while the strength of the relationship over time was uncertain, it was reasonable for the model to include a benefit in OS for patients treated with lenalidomide compared with BSC. Based on the additional submitted evidence [24], the AC also concluded that progression to AML curves should be similar for both lenalidomide and BSC.

\subsubsection{Final Guidance October 2013}

Despite the additional analysis of the manufacturer, the committee did not change the guidance of the first ACD. In the FAD, they concluded that lenalidomide could not be recommended for treating transfusion-dependent anemia caused by low- or intermediate-1-risk MDS associated with del5q cytogenetic abnormality when other therapeutic options were insufficient or inadequate. Given the uncertainties, the committee concluded that the most plausible ICER was above $£ 70,000$ per QALY.

\subsubsection{The Proposed PAS}

The October 2013 FAD for lenalidomide was withdrawn after the submission of an approved PAS by the manufacturer. Under the PAS, the manufacturer would provide lenalidomide at no cost to the NHS for patients with transfusion-dependent low- or intermediate-1-risk MDS with isolated del5q abnormality who continued with lenalidomide treatment beyond 26 cycles. This PAS is similar to the existing PAS of lenalidomide for patients with multiple myeloma. The PAS therefore reduces the long-term drug costs for patients who receive more than 26 cycles of lenalidomide. A revised version of the model was submitted and reviewed by the ERG. The deterministic ICER with the PAS was $£ 25,544$. Minor adjustments to the sensitivity analysis were made by the ERG as these were also made earlier in the ERG-defined base-case. At a threshold of $£ 20,000$ per QALY, $26.2 \%$ of simulations were cost effective and, at a threshold of $£ 30,000,66.6 \%$ of simulations were cost effective. The ERG reviewed the proposed PAS and economic model. No additional issues apart from those stated earlier were identified.

\subsubsection{Preliminary Guidance (Second ACD)}

The main concerns raised by the $\mathrm{AC}$ were uncertainties with regard to the ICER. These uncertainties included patient survival, the proportion of patients eligible for the PAS, and the timing of the PAS rebate. These did not only influence the point estimate but also the cloud of possible outcomes around the ICER. For patient survival, the committee concluded that, despite uncertainty regarding the strength of the relationship, it was reasonable to assume a relationship between transfusion independence and OS. Therefore, it was plausible that lenalidomide indirectly improved survival by reducing transfusion dependence. The committee stated that treatment interruptions were not accounted for in the PAS and that the proportion of people surviving beyond 26 cycles in clinical practice was uncertain. Due to the nature of the PAS, cost reductions were obtained from patients receiving treatment after 26 cycles. If this proportion is uncertain in daily practice, the potential cost savings from the PAS are also subject to uncertainty. As a consequence, the ICER could be much higher. As a response to the concerns related to the PAS, the manufacturer included treatment interruptions, leading to a longer period of time before the PAS comes into effect ( 26 cycles plus 16 days updated the ICER to $£ 25,300$ ). Additional evidence was provided by the manufacturer based on the MDS-004 trial and real-world data that supported the proportion of patients on active treatment currently used in the model $(31.9 \%)$. They also conducted an additional analysis on the proportion of patients eligible for the PAS, i.e. the proportion of patients on active treatment after 26 cycles. This showed that when $27 \%$ or more patients reach 26 cycles of treatment, lenalidomide remains cost effective at a threshold of $£ 30,000$ per QALY.

\subsection{Final Guidance}

After considering the available evidence from the manufacturer, the ERG, expert testimony, and other consultees, the NICE AC decided to recommend lenalidomide for treating low- or intermediate-1-risk MDS associated with an isolated del5q cytogenetic abnormality when other therapeutic options were insufficient or inadequate. The committee agreed that the ICER was uncertain but accepted that a commitment from the manufacturer to publish data on the proportion of patients receiving treatment beyond 26 cycles provided reassurance that lenalidomide was a cost-effective use of NHS resources. 


\section{Conclusions}

The STA presented here describes the first treatment alternative for MDS del5q patients. Clinical evidence was obtained from a single, randomized, phase III trial with a crossover design after 16 weeks. The AC decided to accept lenalidomide as treatment for low- and intermediate-1-risk MDS del5q patients, although the crossover design of the trial as well as the PAS increased the uncertainty of the ICER. A commitment from the manufacturer to collect data provided reassurance that the uncertainties surrounding the ICER can be reassessed when the guidance is reviewed. Nevertheless, the AC stated that if lenalidomide was not a cost-effective use of NHS resources, the foregone health benefits to other NHS patients until the review cannot be regained. This appraisal illustrated that the AC can accept a treatment as cost effective under the acceptance of a commitment of the manufacturer to collect and publish data.

This case study saw a PAS accepted by the Ministry of Health after the initial FAD. While lenalidomide for treating MDS patients with del5q cytogenetic abnormalities was initially not recommended, the PAS reduced the ICER substantially from approximately $£ 68,100$ to $£ 25,300$ per QALY. This changed the recommendation from the AC. The generalizability of the cost-effectiveness results to other countries depends on whether such a PAS is also introduced in these countries, as well as the transferability of underlying utility and survival estimates.

\begin{abstract}
Acknowledgments This project was funded by the National Institute for Health Research (NIHR) Health Technology Assessment (HTA) Program (project number 11/07/01) [see the HTA program website for further information-http://www.hta.ac.uk]. This summary of the ERG report was compiled after the AC's review. The views and opinions expressed are the authors' and do not necessarily reflect those of the HTA Programme, NICE, NIHR, NHS, or the Department of Health. Hedwig Blommestein, Nigel Armstrong, Steve Ryder, Sohan Deshpande, Gill Worthy, Caro Noake, Rob Riemsma, Jos Kleijnen, Johan Severens and Maiwenn Al have no conflicts of interest that are directly relevant to the content of this review.
\end{abstract}

Author contributions Hedwig Blommestein acted as health economist on this assessment, critiqued the manufacturer's economic evaluation, wrote the manuscript and acts as overall guarantor for the overall content of this article. Nigel Armstrong acted as health economist on this assessment and critiqued the manufacturer's economic evaluation. Steve Ryder and Sohan Deshpande acted as systematic reviewers, and critiqued the clinical effectiveness methods and evidence. Gill Worthy acted as statistician and critiqued the analyses in the MS. Caro Noake critiqued the search methods in the submission. Rob Riemsma acted as project lead and systematic reviewer on this appraisal, and critiqued the clinical effectiveness methods and evidence. Jos Kleijnen critiqued the manufacturer's definition of the decision problem and their description of the underlying health problem and current service provision, and acted as supervisor of the project. Johan Severens critiqued the manufacturer's economic evaluation and provided general guidance. Maiwenn Al acted as health economic project lead and critiqued the manufacturer's economic evaluation. All authors contributed to the conception and planning of the work, and critically revised and approved the final submitted version of the manuscript.

Open Access This article is distributed under the terms of the Creative Commons Attribution-NonCommercial 4.0 International License (http://creativecommons.org/licenses/by-nc/4.0/), which permits any noncommercial use, distribution, and reproduction in any medium, provided you give appropriate credit to the original author(s) and the source, provide a link to the Creative Commons license, and indicate if changes were made.

\section{References}

1. Guide to single technology appraisal process. London: NICE; 2009. Available at: http://www.nice.org.uk/Media/Default/About/ what-we-do/NICE-guidance/NICE-technology-appraisals/Guideto-the-single-technology-appraisal-process.pdf. Accessed 30 March 2015.

2. Myelodysplastic syndrome (deletion 5q)—lenalidomide [ID480]. Available at: http://guidance.nice.org.uk/TAG/291. Accessed 30 March 2015.

3. Wade R, Duarte A, Simmonds M, Rodriguez-Lopez R, Duffy S, Woolacott $\mathrm{N}$, et al. The clinical and cost effectiveness of aflibercept in combination with irinotecan and fluorouracil-based therapy (FOLFIRI) for the treatment of metastatic colorectal cancer which has progressed following prior oxaliplatin-based chemotherapy: a critique of the evidence. Pharmacoeconomics. 2015;33(5):457-66.

4. Stevenson M, Pandor A, Stevens JW, Rawdin A, Rice P, Thompson J, et al. Nalmefene for reducing alcohol consumption in people with alcohol dependence: an Evidence Review Group perspective of a nice single technology appraisal. Pharmacoeconomics. 2015;33(8):833-847.

5. Holmes M, Davis S, Simpson E. Alteplase for the treatment of acute ischaemic stroke: a NICE single technology appraisal. An Evidence Review Group perspective. Pharmacoeconomics. 2015;33(3):225-33.

6. Greenhalgh J, Bagust A, Boland A, Dwan K, Beale S, Hockenhull $\mathrm{J}$, et al. Erlotinib and gefitinib for treating non-small cell lung cancer that has progressed following prior chemotherapy (review of NICE technology appraisals 162 and 175): a systematic review and economic evaluation. Health Technol Assess. 2015;19(47):1-134.

7. Latimer NR, Carroll C, Wong R, Tappenden P, Venning MC, Luqmani R. Rituximab in combination with corticosteroids for the treatment of anti-neutrophil cytoplasmic antibody-associated vasculitis: a NICE single technology appraisal. Pharmacoeconomics. 2014;32(12):1171-83.

8. Simpson EL, Davis S, Thokala P, Breeze PR, Bryden P, Wong R. Sipuleucel-T for the treatment of metastatic hormone-relapsed prostate cancer: a NICE single technology appraisal. An Evidence Review Group perspective. Pharmacoeconomics. 2015 [Epub ahead of print].

9. Understanding myelodysplastic syndromes: a patient handbook. 2008. Available at: www.mds-foundation.org/pdf/handbookenglish.pdf. Accessed 30 March 2015.

10. Greenberg PL, Young NS, Gattermann N. Myelodysplastic syndromes. Hematology Am Soc Hematol Educ Program. 2002:136-61.

11. Bowen D, Culligan D, Jowitt S, Kelsey S, Mufti G, Oscier D, et al. Guidelines for the diagnosis and therapy of adult 
myelodysplastic syndromes. $\mathrm{Br} \quad \mathrm{J} \quad$ Haematol. 2003;120(2):187-200.

12. Health and Social Care Information Centre. Hospital episode statistics. Available at: http://www.hesonline.nhs.uk/. Accessed 30 March 2015.

13. Balducci L. Transfusion independence in patients with myelodysplastic syndromes: impact on outcomes and quality of life. Cancer. 2006;106(10):2087-94.

14. Committee for Medicinal Products for Human Use. Assessment report: revlimid. Procedure No. EMEA/H/C/000717/II/0056. EMA/297642/2013. Available at: http://www.ema.europa.eu/ docs/en_GB/document_library/EPAR_-_Assessment_Report_-Variation/human/000717/WC500146340.pdf. Accessed 30 March 2015.

15. Fenaux P, Giagounidis A, Selleslag D, Beyne-Rauzy O, Mufti G, Mittelman M, et al. A randomized phase 3 study of lenalidomide versus placebo in RBC transfusion-dependent patients with low-/ Intermediate-1-risk myelodysplastic syndromes with del5q. Blood. 2011;118(14):3765-76.

16. Jaeger M, Aul C, Sohngen D, Germing U, Schneider W. Secondary hemochromatosis in polytransfused patients with myelodysplastic syndromes. Beitr Infusionsther. 1992;30:464-8.

17. Kontoghiorghes GJ, Pattichi K, Hadjigavriel M, Kolnagou A. Transfusional iron overload and chelation therapy with deferoxamine and deferiprone (L1). Transfus Sci. 2000;23(3):211-23.

18. Wahlin A, Markevarn B, Golovleva I, Nilsson M. Prognostic significance of risk group stratification in elderly patients with acute myeloid leukaemia. Br J Haematol. 2001;115(1):25-33.
19. Szende A, Schaefer C, Goss TF, Heptinstall K, Knight R, Lubbert $\mathrm{M}$, et al. Valuation of transfusion-free living in MDS: results of health utility interviews with patients. Health Qual Life Outcomes. 2009;7:81.

20. Dolan SP, Gudex C, Kind P, Williams A. A social tariff for EuroQol: results from a UK general population survey. The University of York. Discussion paper 138. 1995. http://www. york.ac.uk/che/pdf/DP138.pdf. Accessed 5 March 2015.

21. Malcovati L, Porta MG, Pascutto C, Invernizzi R, Boni M, Travaglino $\mathrm{E}$, et al. Prognostic factors and life expectancy in myelodysplastic syndromes classified according to WHO criteria: a basis for clinical decision making. J Clin Oncol. 2005;23(30):7594-603.

22. Germing U, Lauseker M, Hildebrandt B, Symeonidis A, Cermak J, Fenaux P, et al. Survival, prognostic factors and rates of leukemic transformation in 381 untreated patients with MDS and del(5q): a multicenter study. Leukemia. 2012;26(6):1286-92.

23. Fenaux P, Mufti GJ, Hellstrom-Lindberg E, Santini V, Finelli C, Giagounidis A, et al. Efficacy of azacitidine compared with that of conventional care regimens in the treatment of higher-risk myelodysplastic syndromes: a randomised, open-label, phase III study. Lancet Oncol. 2009;10(3):223-32.

24. Kuendgen A, Lauseker M, List AF, Fenaux P, Giagounidis AA, Brandenburg NA, et al. Lenalidomide does not increase AML progression risk in RBC transfusion-dependent patients with lowor intermediate-1-risk MDS with del(5q): a comparative analysis. Leukemia. 2013;27(5):1072-9. 